Classification

Physics Abstracts

81.15Lm - 61.16d

\title{
Comparison at the microscopic scale of mixed fatty acid-protein Langmuir-Blodgett films resulting from horizontal or vertical transfer
}

\author{
Stéphane Alexandre $\left({ }^{1}\right)$, Nicolas Dubreuil $\left({ }^{1}\right)$, Catherine Fiol $\left({ }^{1}\right)$, Jean-Jacques Malandain $\left({ }^{2}\right)$, \\ Françoise Sommer $\left({ }^{3}\right)$ and Jean-Marc Valleton $\left({ }^{1}\right)$ \\ ( $\left.{ }^{1}\right)$ URA 500 CNRS, UFR des Sciences, Université de Rouen, 76821 Mont-Saint-Aignan, France \\ $\left({ }^{2}\right)$ URA 808 CNRS, UFR des Sciences, Université de Rouen, 76821 Mont-Saint-Aignan, France \\ $\left({ }^{3}\right)$ CENATS, Université Claude Bernard, Lyon I, 43 Boulevard du 11 Novembre 1918, 69622 \\ Villeurbanne, France
}

(Received July 4; accepted October 26, 1994)

\begin{abstract}
Résumé. - Des films mixtes de Langmuir associant acide béhénique et glucose oxydase ont été transférés par des techniques de transfert horizontal ou de transfert vertical sur graphite pyrolytique. La structure de ces films a été comparée par microscopie électronique à balayage et par microscopie à force atomique aux échelles microscopique et nanoscopique. Les structures observées à l'échelle du micromètre sont assez différentes pour les deux types de transfert (homogénéité, tailles caractéristiques, orientations). L'arrangement des molécules d'enzyme est toutefois comparable à l'échelle submicromètrique dans les deux cas.
\end{abstract}

\begin{abstract}
Mixed Langmuir films in which behenic acid and glucose oxidase are associated have been transferred by vertical or horizontal lifting methods onto pyrolytic graphite. The resulting films have been compared by scanning electron microscopy and by scanning force microscopy. The structures observed at the microscopic scale are different for the two transfer techniques in terms of homogeneity, dimensions and orientations. At a nanoscopic scale however, the organization of enzyme molecules is quite analogous in both cases.
\end{abstract}

\section{Introduction.}

The incorporation of biomolecules (enzymes, antibodies, lectins ...) in well defined structures is an ambitious goal; the interest of this approach is twice: on the one hand, such structures may constitute interesting models for biological membranes and may be used for studying complex functions in a simplified structure. On the other hand, such structures may be used in the field of biomimetics, in order to design artificial systems inspired by biological structures and/or functions, and possessing analogous properties; among them biosensing is a major target [1-3]. 
Langmuir-Blodgett (LB) technology plays a privileged role in this field since it allows the elaboration of three dimensional ordered structures by using amphiphilic long chain molecules such as fatty acids or lipids which are the constituents of natural membranes [4, 5].

In order to design a biosensor by LB technology it is necessary to associate an amphiphilic molecule which will constitute the matrix and a biomolecule which will give to the system the specificity of recognition of particular chemical or biochemical substances [6-12]. The biosensors based on LB technology utilize an active layer constituted of one or several layers of the amphiphilic molecule, in which the active biomolecule is incorporated. Frequently, the biomolecule involved in these systems is an enzyme. The major interest of LB technology is to create an adequate organization of the two components at the molecular scale; in particular the very small thickness of the structures elaborated leads to biosensors with very short response times.

Scanning force microscopy plays a privileged role in the characterization of LB films [13-19]. Scanning force microscopy, with its different possibilities, is a unique tool for imaging these systems at a microscopic or at a molecular level. Few papers, however, dealing with imaging of mixed layers of proteins and fatty acids have been published [20-26].

In a previous paper [26], we studied by scanning force microscopy mixed Langmuir-Blodgett films constituted of behenic acid (BA) and glucose oxidase (GOx) transferred on HOPG by a horizontal lifting method; the images obtained showed, at a molecular level, the arrangement of enzyme molecules in 2D crystal structures. However at the micrometer scale, the films are characterized by a high heterogeneity. It was also possible to distinguish the individual structure of the protein revealing the two subunits. On top of these molecules, behenic acid molecules were observed. In addition, we presented a model of the structure consistent with the images and quantitative data (height profiles) obtained by this technique.

In this paper, we present new results concerning the comparison of the structure of mixed BAGOx LB films transferred on HOPG by two different techniques: the horizontal lifting method used in our previous work, and the more classical technique of vertical transfer in which the sample goes vertically through the interface. Scanning force microscopy was the privileged technique used for this study; scanning electron microscopy was also used in order to characterize the sample at a submillimeter scale.

\section{Materials and methods.}

A Langmuir-Blodgett trough ATEMETA (Paris) was utilized for the preparation of the samples; the dimensions of the trough are $50 \mathrm{~cm} \times 7 \mathrm{~cm}$; the volume of the liquid phase is $250 \mathrm{~cm}^{3}$. This system uses a mobile barrier for compressing the amphiphilic molecules and a Wilhelmy balance for measuring the interfacial pressure. The temperature of the subphase was $22^{\circ} \mathrm{C}$ and the system operated in the air.

For the elaboration of the mixed layer of behenic acid and glucose oxidase, an aqueous solution of glucose oxidase was used as subphase, and a behenic acid solution in chloroform was spread on its surface. Aqueous solutions were prepared with water obtained by a Millipore system involving deionization, reverse osmosis and filtration. Glucose oxidase was obtained from SIGMA (type VII; Aspergillus niger) and used without further purification. Its concentration in the subphase was $3.2 \mathrm{mg} / \mathrm{l}$. Behenic acid (SIGMA, purity: $99 \%$ ) was prepared as a $10^{-3} \mathrm{M}$ solution in chloroform, and an amount of $0.1 \mathrm{ml}$ was spread with a micropipette. In order to obtain reproducible injections of behenic acid samples, a system was designed for positionning the pipette vertically, at a constant height above the interface.

After spreading the behenic acid solution, the enzyme molecules were allowed to adsorb onto the polar heads of behenic acid molecules for $30 \mathrm{~min}$. Then the behenic acid molecules were compressed upto $30 \mathrm{mN} / \mathrm{m}$. 
After the compression, the film was transferred either by the horizontal lifting method or by the vertical method. Horizontal and vertical transfers were realized on HOPG samples (highly oriented pyrolytic graphite) obtained from Le Carbone Lorraine (Paris); HOPG was cleaved before any transfer, and used without any particular processing.

For the vertical transfer, the feed-back loop (control of the mobile barrier by the signal produced by the Wilhelmy balance) was kept closed. HOPG samples, initially in the air, were covered by two layers by a down and up displacement through the interface. The rate of transfer is $1 \mathrm{~cm} / \mathrm{min}$.

For the horizontal transfer, the feedback loop was also kept closed and a system with two articulations was used in order to keep the sample as horizontal as possible. However, in spite of this device, the transfer of the second layer constituting the bilayer formed by the horizontal transfer was quite asymmetrical $[27,28]$. The fact that a mixed bilayer system was formed by the horizontal transfer process was confirmed by transfer records. The horizontal lifting method has the particularity of producing samples which are completely covered by the Langmuir-Blodgett films, which facilitates the analysis by scanning force microscopy.

Scanning electron microscopy was performed with a JEOL JSM35CF microscope; the tension used was $20 \mathrm{kV}$, and the magnification rate was kept to the low value of 200 for the images presented in this paper; for higher magnifications, some damage is caused to the LB films. In order to minimize these damages, a very low current was used. No special preparation was used for examining LB films.

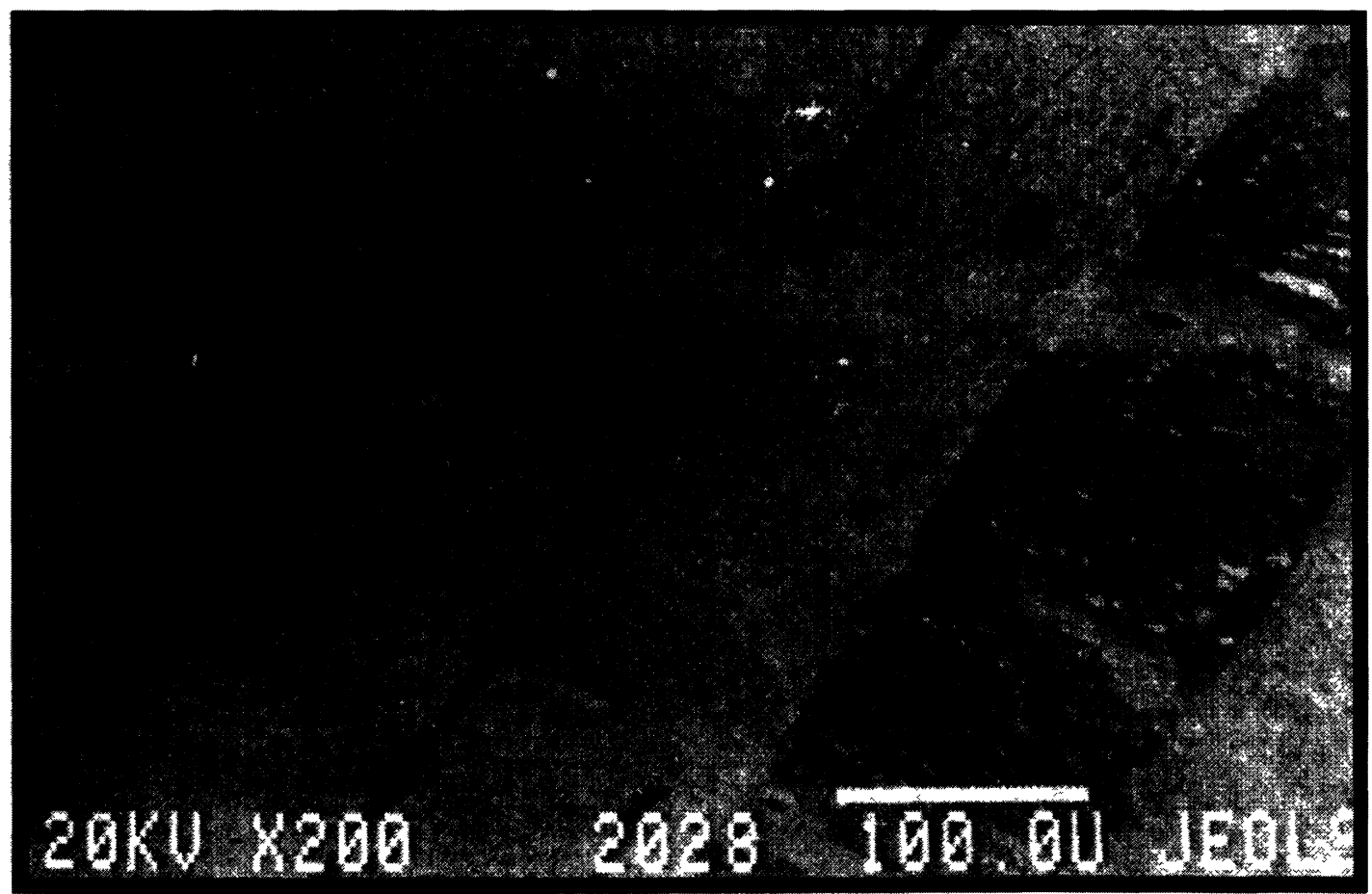

Fig. 1. - Scanning electron microscopy image $(500 \mu \mathrm{m} \times 350 \mu \mathrm{m})$ of a mixed bilayer constituted of behenic acid and glucose oxidase transferred on HOPG by a vertical lifting technique; parallel defects are clearly visible in the film. 
SFM experiments realized were either contact mode or "tapping" mode experiments.

Contact mode measurements were performed with a Nanoscope II model from Digital Instruments (Santa Barbara, CA, USA) with a $140 \mu \mathrm{m}$ scanner for large range analysis, and with a $1 \mu \mathrm{m}$ scanner for molecular resolution studies. Long range measurements were achieved in the air, whereas molecular resolution measurements were achieved in water by using the "liquid cell". All the measurements were performed with the feed-back loop on (constant force: $10^{-9}$ to $10^{-8} \mathrm{~N}$ ) On long range images, no processing was used except a flatten operation used for obtaining the horizontality of graphite terraces. On high resolution images, in addition to the flatten operation, FFT filtering operations were made in some cases.

"Tapping" mode measurements were performed with a Nanoscope III model from Digital Instruments (Santa Barbara, CA, U.S.A.) with $14 \mu \mathrm{m}$ and $140 \mu \mathrm{m}$ scanners, cantilevers were made of silicon; their spring constant was about $50 \mathrm{~N} / \mathrm{m}$; they operated at an oscillation frequency of $370 \mathrm{kHz}$; the amplitude of oscillations was determined by voltages between 80 and $120 \mathrm{mV}$.

All the images are presented in height mode (palette of colour for height: dark colours for low zones, light colours for high zones), and are 2D images.

\section{Results.}

3.1 SCANNING ELECTRON MICROSCOPY. - Scanning electron microscopy images (Figs. 1 and 2) reveal a quite different structure for the mixed BA/GOx films produced by horizontal and vertical

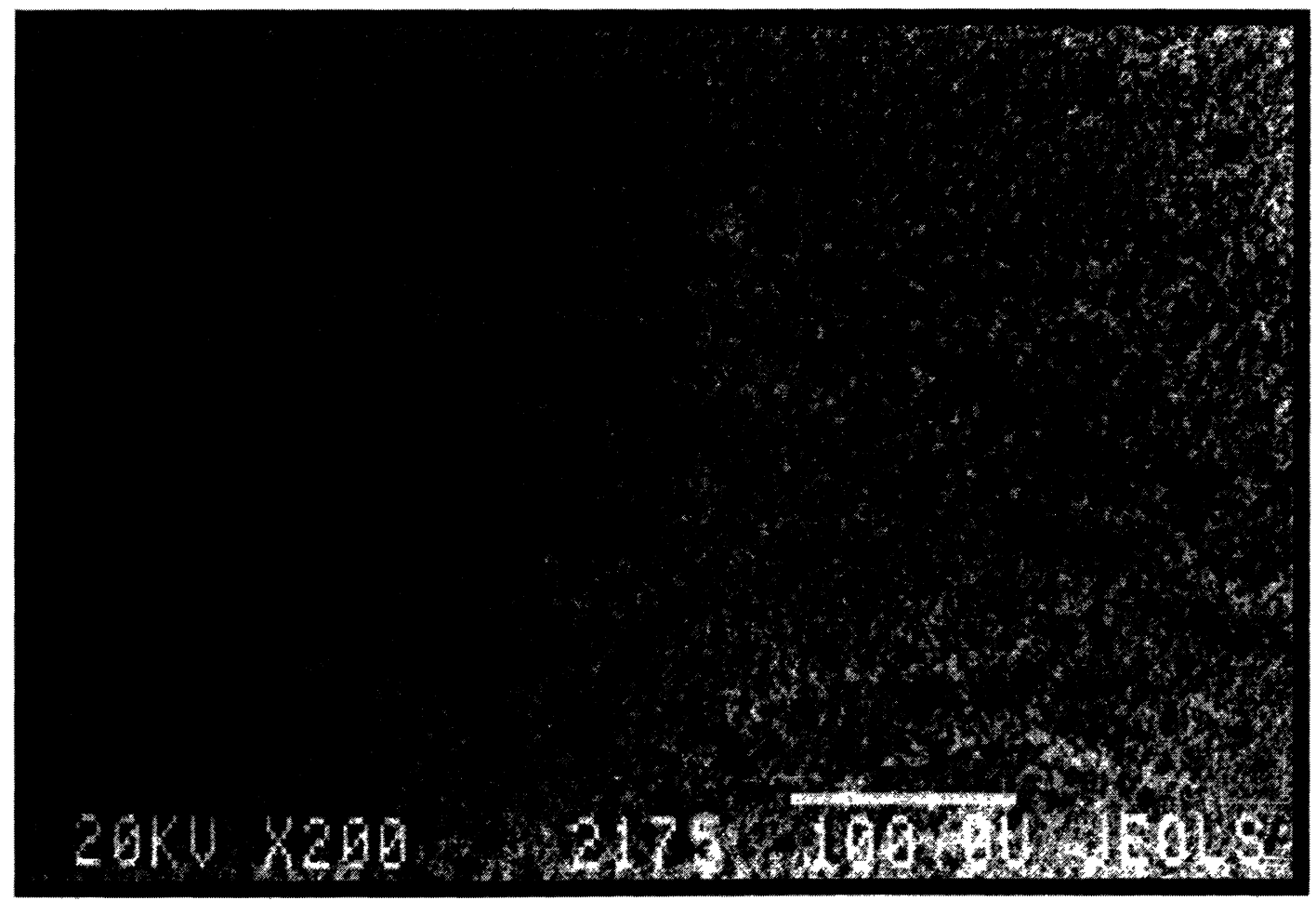

Fig. 2. - Scanning electron microscopy image $(500 \mu \mathrm{m} \times 350 \mu \mathrm{m})$ of a mixed bilayer constituted of behenic acid and glucose oxidase transferred on HOPG by a horizontal lifting technique; the structure appears more isotropic than the one observed in the case of the vertical transfer. 
transfers.

For vertical transfers (Fig. 1), the films are characterized by parallel defects corresponding apparently to an absence of film, which might explain the contrast observed: the dark parts in the image of figure 1 might correspond to the graphite surface, whereas the lighter parts might correspond to a less conducting material, the LB film. These defects are parallel to a direction which corresponds to the direction of the line intersection of the substrate with the air/water interface during the transfer process. In addition, these parallel defects are not homogeneous in terms of width, and it seems that the defects are all the more important than the zone observed is close to the upper limit of the film transferred. This type of defect is probably due to the transfer step; moreover the interactions between the tails of BA molecules and graphite are weak and these defects might be interpreted as the result of a partial "sliding" of the film on the graphite surface. It is also possible to observe other types of defects in these films: holes of different sizes and shapes, small aggregates and small parts of film superposed on an almost continuous film.

For horizontal transfers, the structure of the films transferred is quite different: they are characterized by a relative homogeneity and isotropy (Fig. 2). However, many defects randomly dispersed appear. Whereas the vertical transfer leads inherently to a privileged direction (parallelism of the large defects), the horizontal transfer leads globally to a more isotropic film. Locally
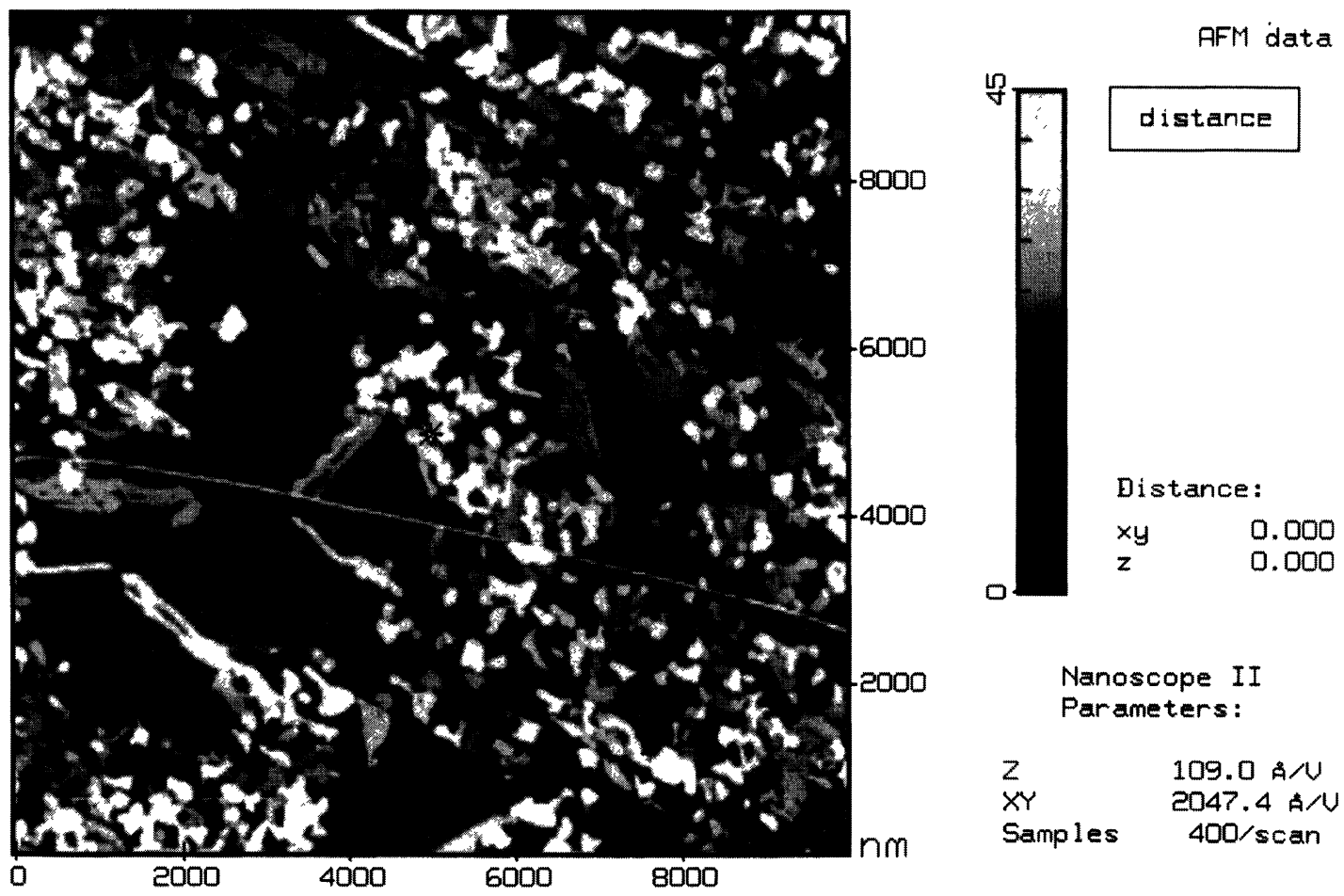

$A B+$ GOx LB FILM (HDR. TRANSF, ) on HOPG

Data taken Fri Feb 12 17:08:40 1993

Buffer 5(FHTRHA3F), Rotated $0^{*}, X Y$ axes [nm], $Z$ axis [nm]

Fig. 3. - Scanning force microscopy image $(10 \mu \mathrm{m} \times 10 \mu \mathrm{m})$ of the mixed bilayer constituted of behenic acid and glucose oxidase transferred on HOPG by the horizontal transfer technique; glucose oxidase island structures are clearly visible. Image obtained in contact mode. 
however (scale of 10 micrometers), the film produced by the vertical transfer seems more homogeneous; this may be due to the direction of the forces interacting between the tails of the fatty acid and the graphite surface during the transfer: quasi tangent in the case of the vertical transfer because of the meniscus, and quasi perpendicular in the case of the horizontal transfer.

Images (not shown here) obtained at a magnification of 10000 show in both cases a good local order with pinholes characterized by a dimension of the order of the tenth of micrometer. These latter results have to be considered with caution, because of the risks of damage at this magnification; it seems, however, that the conclusion of this SEM investigation might be that at a large scale (10 to 100 micrometers) vertical and horizontal transfers lead to very different structures and defects; at a lower scale, both lead to a more comparable structure. This assessment has been confirmed by scanning force microscopy.

3.2 SCANNING FORCE MICROSCOPY; A MICROSCOPIC STUDY. - In order to study the microscopic structure of the mixed BA/GOx films, scanning force microscopy has been utilized in two different modes: the classical contact mode and the "tapping" mode; this parallel study has been used for'validating the two modes for these soft samples and comparing the results obtained when one mode or the other is used. The "tapping" mode is known for avoiding to cause damage to
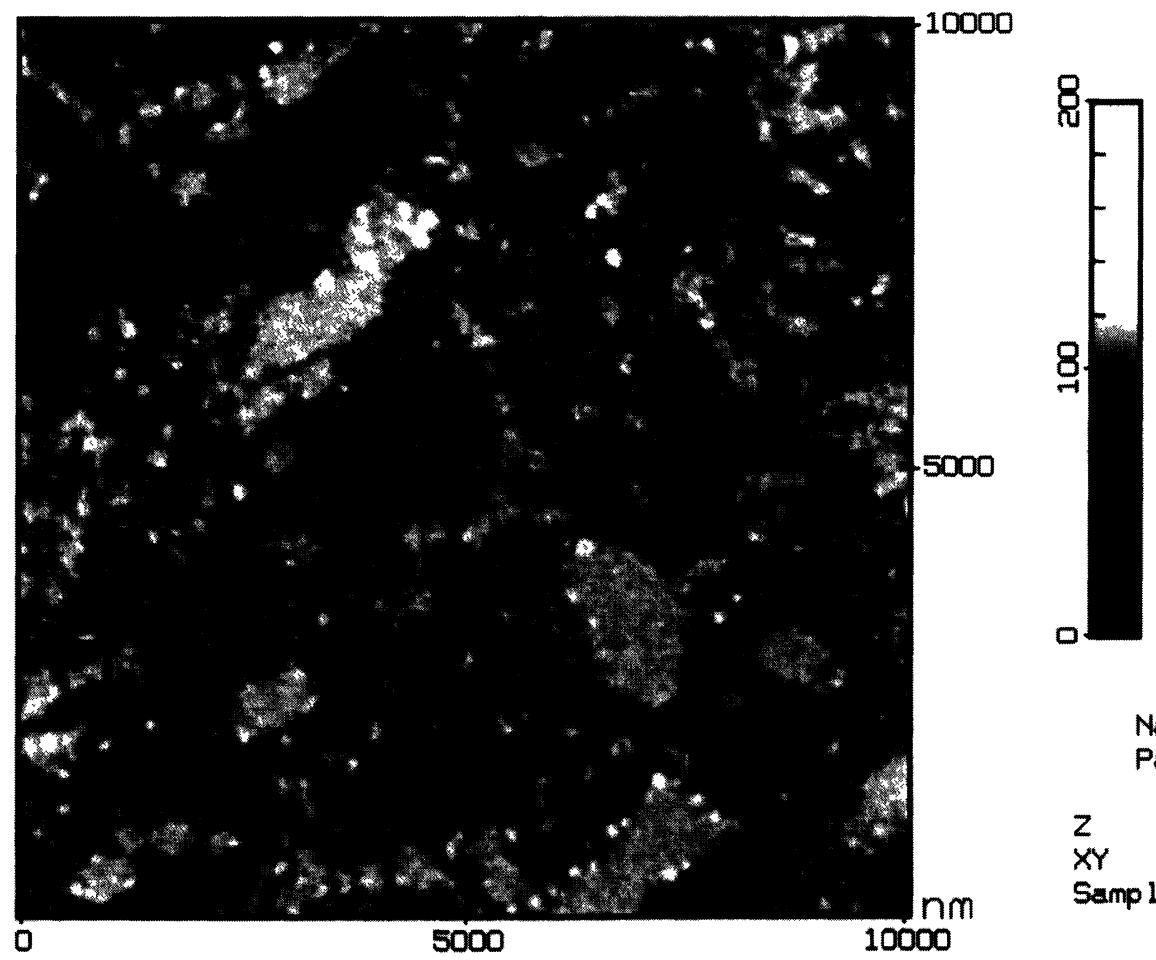

AFM data

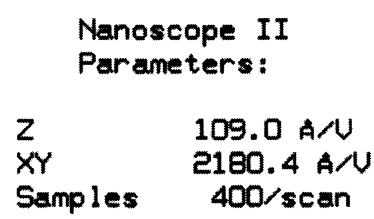

BA + GOx on HOPG Vert. Transf.

Data taken Wed Jun 09 14:20:15 1993

Buffer 3 (abgo.t(Z)), Rotated $0^{\circ}, X Y$ axes [nm], $Z$ axis [nm]

Fig. 4. - Scanning force microscopy image $(10 \mu \mathrm{m} \times 10 \mu \mathrm{m})$ of the mixed bilayer constituted of behenic acid and glucose oxidase transferred on HOPG by the vertical transfer technique; the organization of glucose oxidase structures is less important than in the case of the horizontal transfer. Image obtained in contact mode. 
soft samples; the classical contact mode authorizes higher resolutions; the two modes have been compared at the micrometer scale, in the range where they can be used.

3.2.1 Contact mode. - At the microscopic scale, SFM measurements reveal a great heterogeneity of the sample obtained by horizontal transfer [26] (image $10 \mu \mathrm{m} \times 10 \mu \mathrm{m}$ of Fig. 3) characterized by randomly dispersed "islands" which have been attributed to enzymatic structures. The coverage ratio by enzyme molecules is approximately $35 \%$. The size dispersity of enzymatic structures is important; the minimum characteristic length observed is about $50 \mathrm{~nm}$, which corresponds approximately to an island of 100 molecules; the largest islands occupy a surface of nearly $1 \mu \mathrm{m}^{2}$; the mean value is about $0.1 \mu \mathrm{m}^{2}$. It is to note that these islands have a well delimited shape and that between them no other structure appears at this scale.

An image of the sample prepared by vertical transfer (Fig. 4) at the same scale $(10 \mu \mathrm{m} \times 10 \mu \mathrm{m})$ appears quite different. The structures observed on this image are characterized by a greater heterogeneity of their dimensions which may reach several $\mu \mathrm{m}^{2}$. In this case it is more difficult to estimate the coverage because of the heterogeneity observed. It is also possible to notice on this image a privileged direction (from the lower left corner to the upper right corner); it seems difficult to relate these alignments to the defects observed in SEM; these alignments may be also related to steps in the graphite structure.

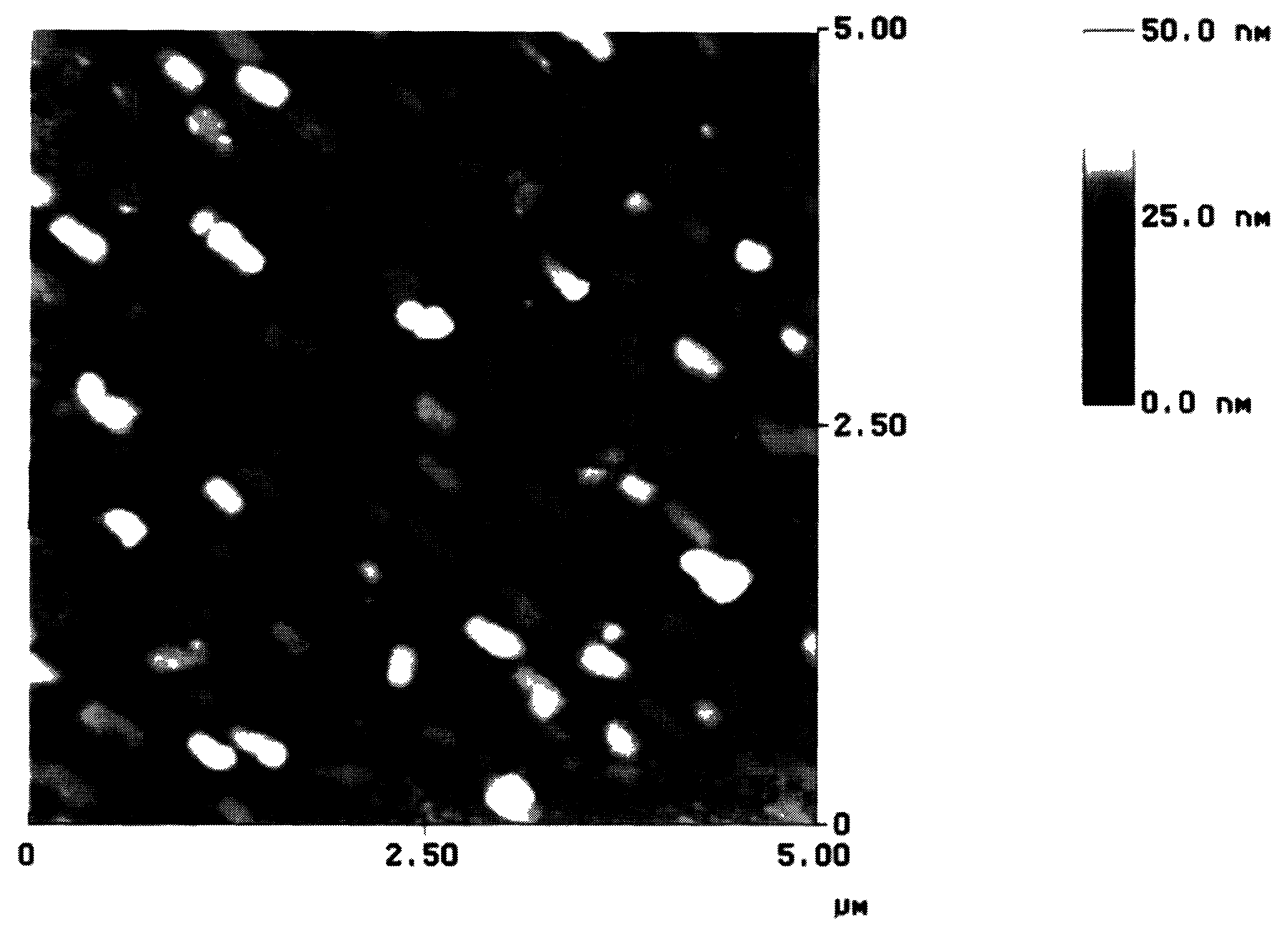

Fig. 5. - Scanning force microscopy image $(5 \mu \mathrm{m} \times 5 \mu \mathrm{m})$ of the mixed bilayer constituted of behenic acid and glucose oxidase transferred on HOPG by the horizontal transfer technique; this image obtained with the tapping mode is analogous to the corresponding images obtained with the contact mode; in addition smaller structures appear between the main structures. 

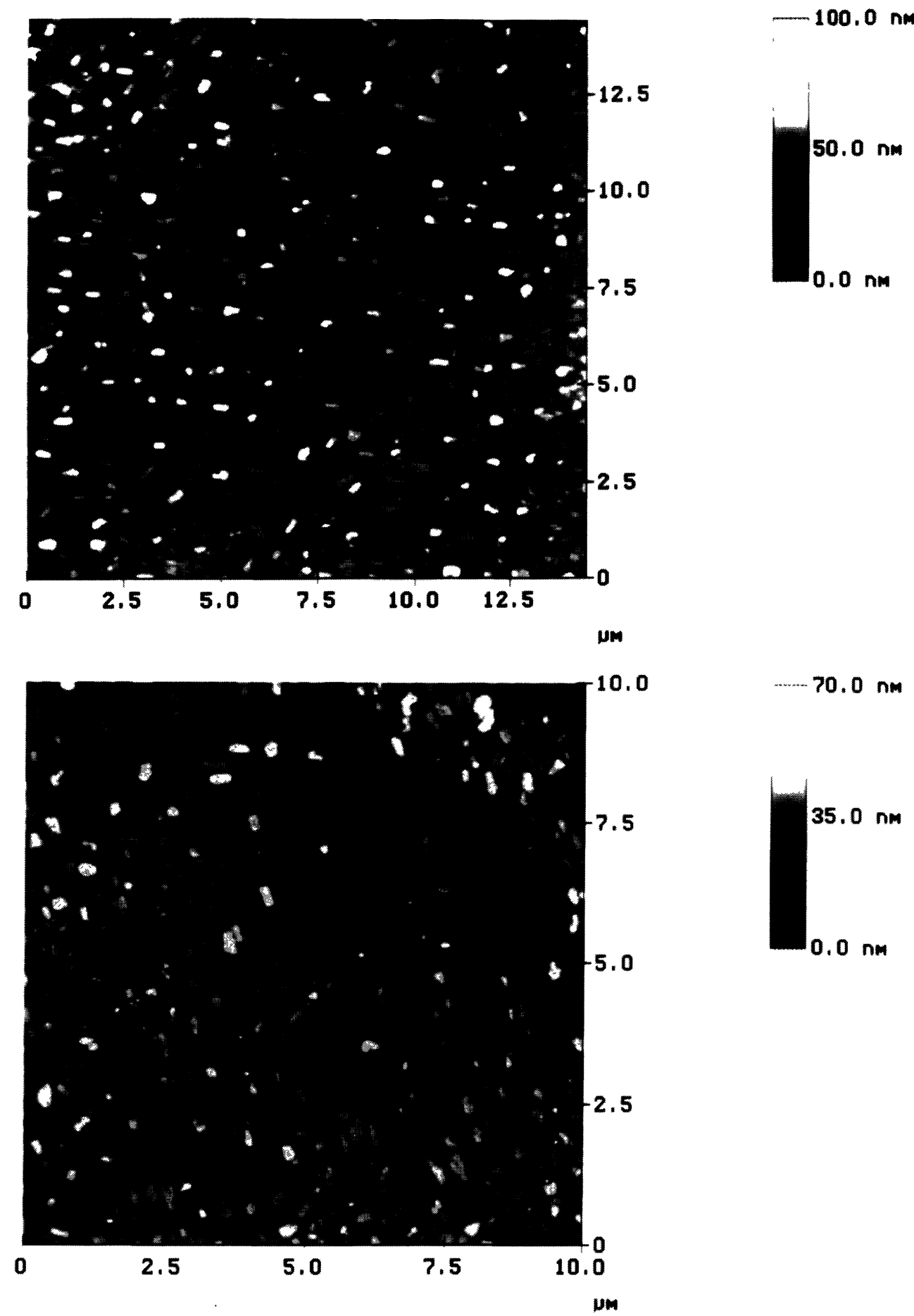

Fig. 6. - Study of reproducibility of the horizontal transfer technique. Scanning force microscopy images obtained with the tapping mode of the mixed bilayer constituted of behenic acid and glucose oxidase transferred on HOPG by the horizontal transfer technique; figure $6 \mathrm{a}(15 \mu \mathrm{m} \times 15 \mu \mathrm{m})$ and figure $6 \mathrm{~b}(10 \mu \mathrm{m} \times 10 \mu \mathrm{m})$ are quite similar. 


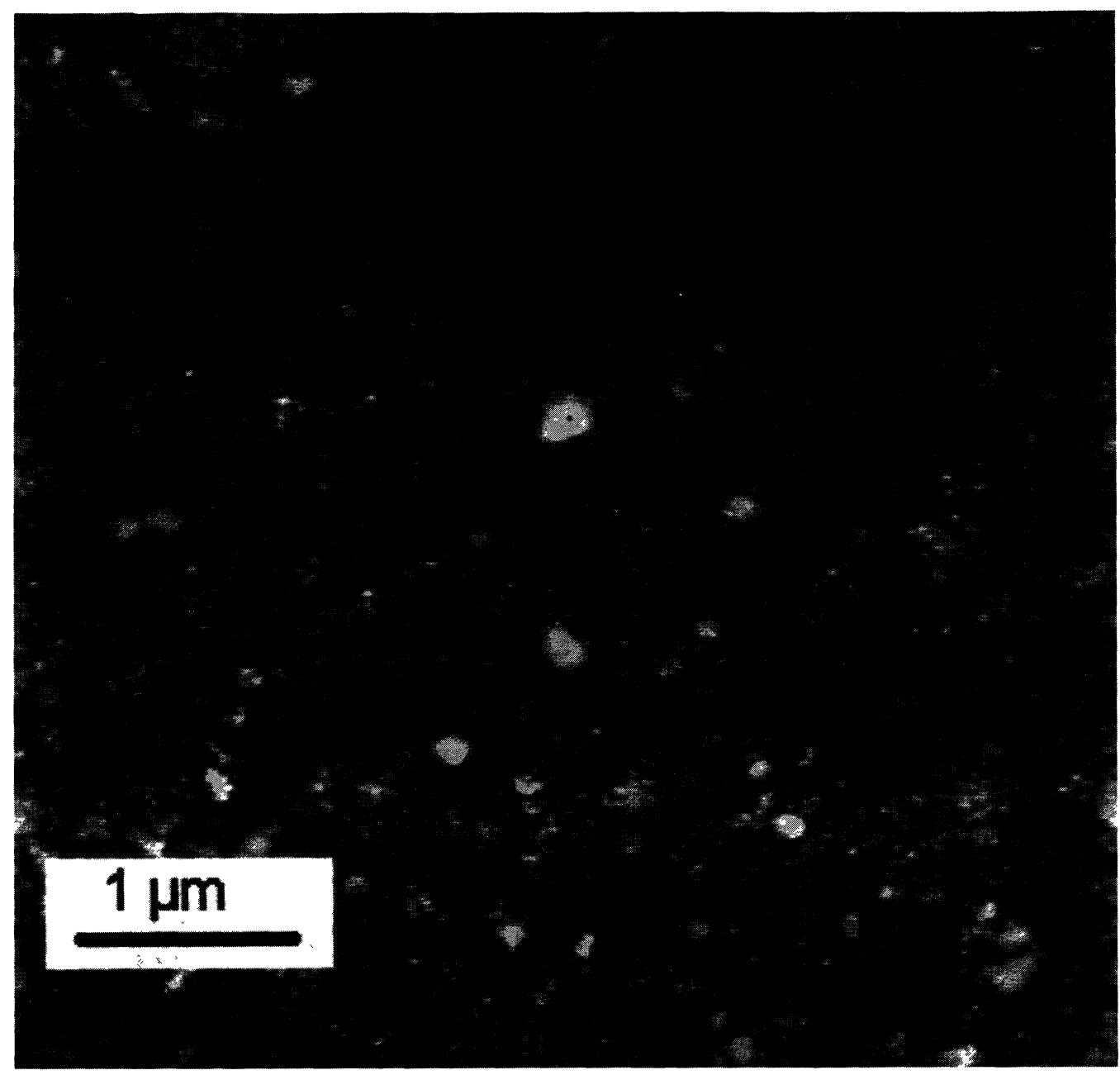

Fig. 7. - Scanning force microscopy image $(5 \mu \mathrm{m} \times 5 \mu \mathrm{m})$ of the mixed bilayer constituted of behenic acid and glucose oxidase transferred on HOPG by the vertical transfer technique; this image obtained with the tapping mode is very similar to the corresponding images obtained with the contact mode.

3.2.2 "Tapping mode". - The images of the sample obtained by horizontal transfer are similar to those obtained by the contact mode: discrete structures appear homogeneously distributed on the surface (Fig. 5); the order seems greater on this image than the order observed by the contact mode; this may be due to the use of the "tapping" mode less agressive for the sample, or to a structure slightly different of the two samples. An estimate of the mean area of the enzyme structures leads to a similar value of $0.1 \mu \mathrm{m}^{2}$. The structures observed with the "tapping" mode have also a more regular quasi rectangular shape, and seem oriented along a privileged direction (from the upper left corner to the lower right corner on this image). In addition to these structures, between them, smaller structures are clearly visible, which were not observed with the contact mode; the same assumptions as the ones exposed for explaining the greater order observed with the "tapping" mode may hold. 

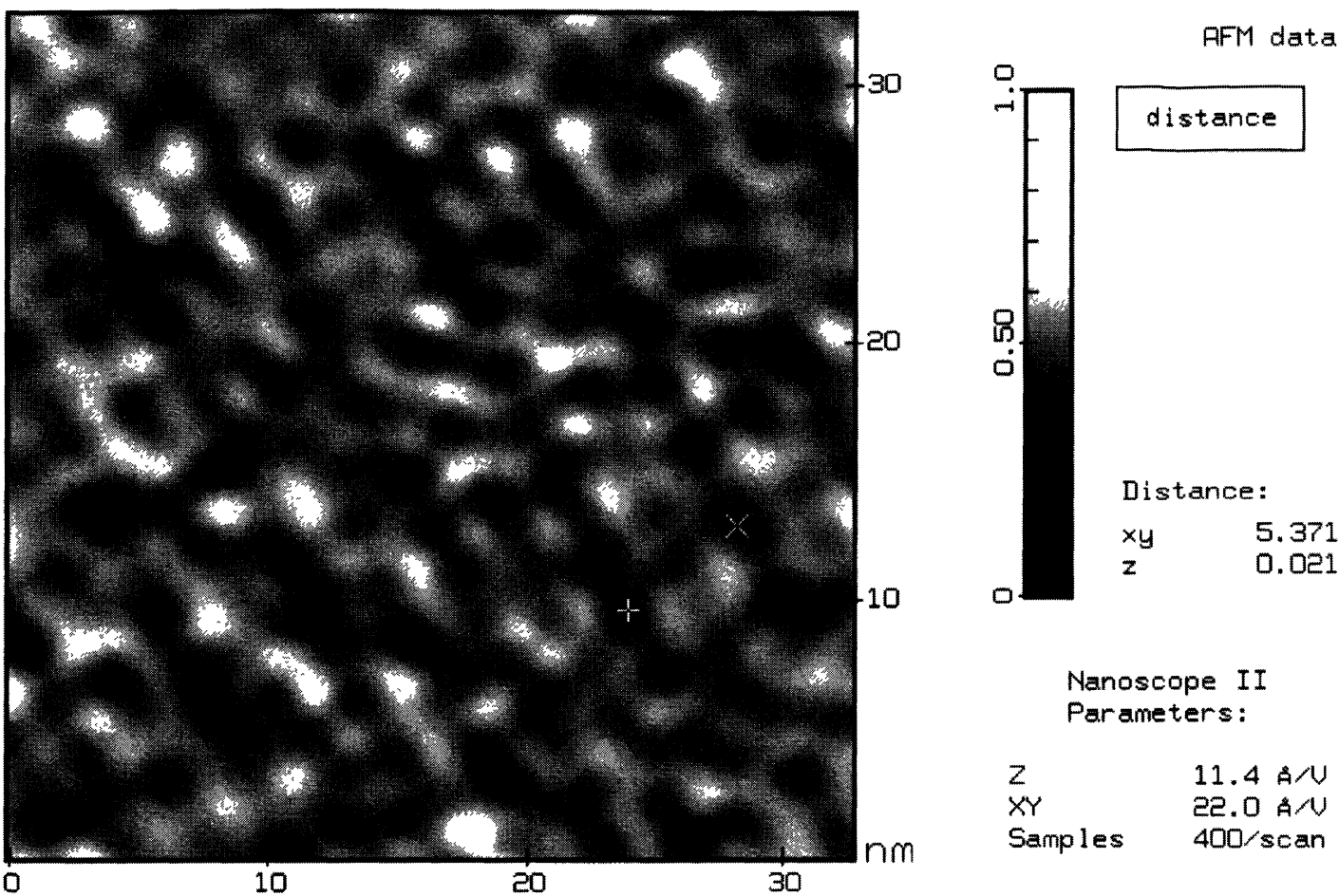

$\mathrm{BA}+\mathrm{GOx}$ on HOPG (H. Tr. Liq. Cel1)

Data taken Mon Mar 01 11:15:20 1993

Buffer 4(3M02.502(Z)), Rotated $0^{\circ}$, XY axes [nm], $Z$ axis [nm]

Fig. 8. - SFM image $(33 \mathrm{~nm} \times 33 \mathrm{~nm})$ of the mixed bilayer constituted of behenic acid and glucose oxidase transferred on HOPG by the horizontal transfer technique, obtained after a FFT processing and filtering; the alignments are attributed to enzymatic structures.

In order to study the reproducibility of the horizontal transfer procedure, other samples were prepared and imaged with the "tapping" mode. The images obtained are very similar (Fig. 6a for a first sample (the same as in Fig. 5); Fig. $6 \mathrm{~b}$ for a second sample); the same rectangular structures appear, with the same dimensions and the same homogeneity; however, the second sample is characterized by the absence in the zone observed of a privileged direction; in addition, the smaller structures observed on the first sample can be seen on the second sample only in a few zones. In spite of these differences, it may be concluded that the reproducibility of the horizontal transfer procedure is quite satisfactory.

Images of the sample prepared by vertical transfer observed by the contact mode and by the "tapping" mode are quite similar; the same structures appear (Fig. 7) with analogous sizes and alignments along a privileged direction can also be seen clearly.

3.3 SCANNING FORCE MICROSCOPY; A NANOSCOPIC STUDY. - The stuctures of the mixed BA/GOx films appear quite different at the micrometer scale, when prepared by a horizontal transfer or by a vertical transfer procedure. The next step has been to compare the organization of these systems at the nanometer scale and we focussed on the main structures which have been attributed to the presence of the enzyme [26]. 


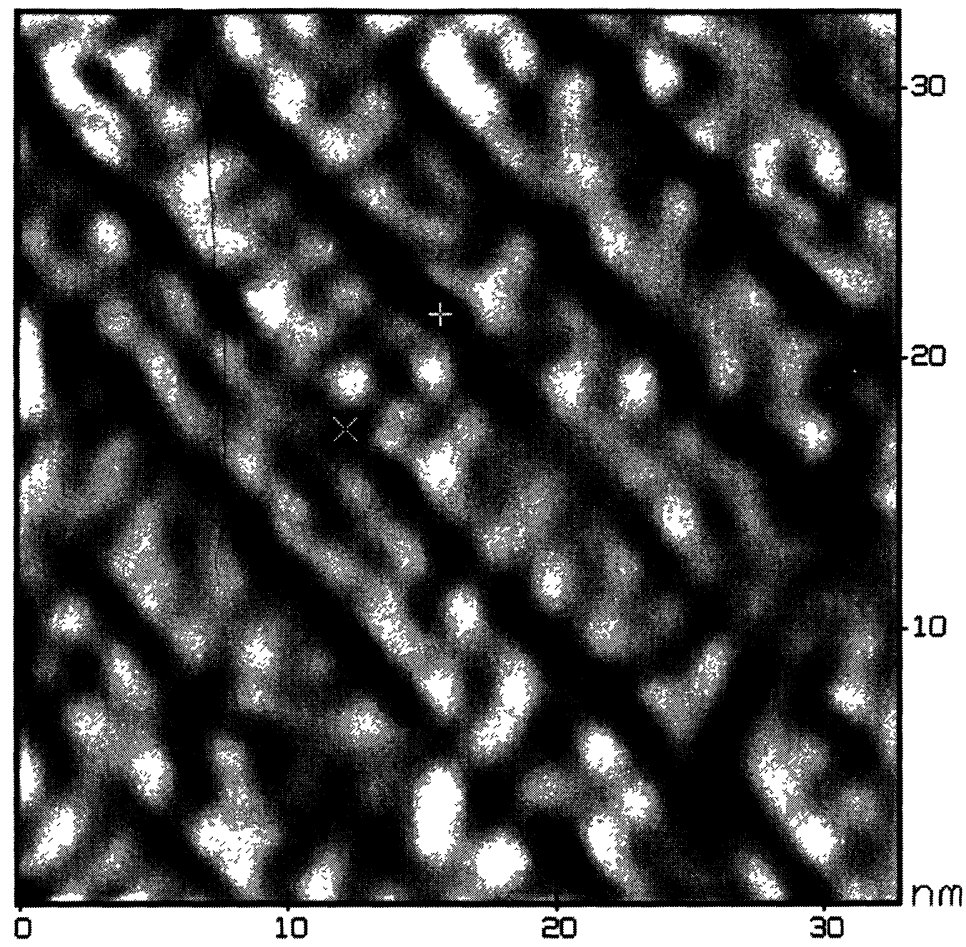

AFM data

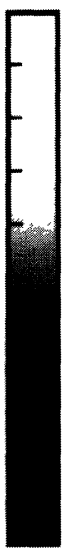

distance

Distance:

$x y$

5.498

$\mathbf{z}$

0.035

Nanoscope II

Parameters:

$Z$

$11.4 \mathrm{~A} / \mathrm{V}$

$x r$

$25.9 \mathrm{~A}$

Samples $400 / \operatorname{scan}$

$B A+$ GOx on HOPG Vert. Transf.

Data taken Tue Jun 15 13:05:43 1993

Buffer 1(GTUJMZTF.14(F)), Rotated $0^{\circ}, X Y$ axes [nm], $Z$ axis [nm]

Fig. 9. - SFM image $(33 \mathrm{~nm} \times 33 \mathrm{~nm})$ of the mixed bilayer constituted of behenic acid and glucose oxidase transferred on HOPG by the vertical transfer technique, obtained after a FFT processing and filtering; the organization is quite similar to the one observed with the horizontal transfer procedure.

For the two transfer techniques, the organization of the enzyme molecules is quite similar: this organization is characterized by parallel alignments of the enzyme molecules, the different alignments constituting a 2D crystal (Fig. 8 for the horizontal transfer, Fig. 9 for the vertical transfer). In the alignments the individual molecules can be seen. In both cases, a depression is visible in the enzyme molecules constituted of two subunits [29]. In the case of the vertical transfer the quality of the images obtained is better (the individual structure appears unambiguously, with a clear division of the molecules in two subunits); but at this scale, obtaining good quality images is rather tricky, and no definite conclusion can be made from this observation.

\section{Conclusion.}

Mixed Langmuir-Blodgett films constituted of behenic acid and glucose oxidase have been transferred on HOPG by vertical and horizontal lifting techniques. A comparative study of the structure of the transferred films have been made by scanning force microscopy and scanning electron microscopy in order to try to determine an optimal protocol.

At the submillimeter scale, the images obtained by SEM studies show a quite different structure; the samples obtained by vertical transfer exhibit large parallel defects, whereas the samples 
obtained by horizontal transfer seem more isotropic.

At the microscopic scale, the images obtained by scanning force microscopy show also different structures for the two transferring techniques; the horizontal transfer technique produces more discrete and regular structures whereas the vertical transfer technique leads to less organized structures. The classical contact mode and the "tapping" mode lead to similar results.

At the molecular level, the arrangement of enzyme molecules in the observed structures appears quite similar for the two types of transfer; a $2 \mathrm{D}$ crystal organization is observed and the individual structure of the enzyme molecules can be seen showing unambiguously the two subunits of the protein.

This work reveals the high heterogeneity of the mixed films constituted of behenic acid and glucose oxidase at the different levels of organization, except at the molecular scale where the structure of the enzymatic aggregates is certainly determined for a great part by a crystal growth process. This conclusion is independent of the type of transfer utilized for the elaboration of the mixed films.

After the study of the structures obtained after a transfer process, it is necessary to study very precisely the elaboration of the mixed fatty acid - protein films at the air/water interface to determine their structure before their transfer on a substrate. This work is under way in the group; it is based on interfacial pressure/area isotherms, stability studies, surface potential measurements and Brewster angle microscopy.

\section{References}

[1] Lowe C.R., Philos. Trans. R. Soc. London Ser. B 324 (1989) 487.

[2] Wang J., Anal. Chem. 63 (1991) R235.

[3] Janata J., Anal. Chem. 64 (1992) R196.

[4] Roberts G., Langmuir-Blodgett Films (Plenum ed., 1990).

[5] Barraud A., J. Chim. Phys. 82 (1985) 683.

[6] Sriyudthsak M., H. Yamagishi and T. Moriizumi, Thin Solid Films 160 (1988) 463.

[7] K. Owaku, H. Shinohara, Y. Ikariyama and M. Aizawa, Thin Solid Films 180 (1989) 61.

[8] J. Anzai, S. Lee and T. Osa, Chem. Pharm. Bull. 37 (1989) 3320.

[9] W. Schumann, S.P. Heyn and H.E. Gaub, Adv. Mater. 3 (1991) 388.

[10] Arisawa S., Arise T. and. Yamamoto R, Thin Solid Films 209 (1992) 259.

[11] Turko I.V., Lepesheva G.I. and Chashchin V.D., Anal. Chim. Acta 265 (1992) 21.

[12] Fiol C., Valleton J.M., Delpire N., Barbey G., Barraud A. and Ruaudel-Teixier A., Thin Solid Films 210-211 (1992) 489.

[13] Weisenhorn A.L., Egger M., Ohnesorge F., Gould S.A.C., Heyn S.P., Hansma H.G., Sinsheimer R.L., Gaub H.E. and Hansma P.K., Langmuir 7 (1991) 8.

[14] Alves C.A., Smith E.L. and Porter M.D., J. Am. Chem. Soc. 114 (1991) 1222.

[15] Meyer E., Overney R., Brodbeck D., Howald L., Luthi R., Frommer J. and Guntherodt H.J., Phys. Rev. Lett. 69 (1992) 1777.

[16] Zasadzinski J.A., Viswanathan R., Madsen L., Garanes J. and Schwartzd. K., Science 263 (1994) 1726.

[17] Bourdieu L., Ronsin O. and Chatenay D., Science 259 (1993) 798.

[18] Peltonen J.P.K., He P. and Rosenholm J.B., Langmuir 9 (1993) 798.

[19] Chi L.F., Eng L.M., Graf K. and Fuchs H., Langmuir 8 (1992) 2255.

[20] Egger M., Ohnesorge F., Weisenhorn A.L., Heyn S.P., Drake B., Prater C.B., Gould S.A.C., Hansma P.K. and Gaub H.E., J. Struct. Biol. 1003 (1990) 89. 
[21] Weisenhorn A.L., Drake B., Prater C.B., Gould S.A.C., Hansma P.K., Ohnesorge F., Egger M., Heyn S.P. and Gaub H.E., Biophys. J. 58 (1990) 1251.

[22] Fiol C., Alexandre S., Delpire N. and Valleton J.M., Thin Solid Films 215 (1992) 88.

[23] Fujiwara I., Ohnishi M. and Seto J., Langmuir 8 (1992) 2219.

[24] Ohnishi S., Hara M., Furuno T. and Sasabe H., Biophys. J. 63 (1992) 1415.

[25] Liu Z.F., Manivannan A., Inokuchi H. and Yanagi H., J. Vac. Sci Technol. B 11 (1993) 1766.

[26] Alexandre S., Dubreuil N., Fiol C. and Valleton J.M., Microsc. Microanal. Microstruct. 5 (1994) 61.

[27] Lee S., Virtanen J.A., Virtanen S.A. and Penner R.M., Langmuir 8 (1992) 1243.

[28] Okahata Y., Ariga K. and Tanaka K., Thin Solid Films 210/211 (1992) 702.

[29] Hecht H.J., Kalisz H.M., Hendle J., Schmid R.D. and Schomburg D., J. Biol. Mol. 229 (1993) 153. 